\title{
BMJ Open Understanding how self-management interventions work for disadvantaged populations living with chronic conditions: protocol for a realist synthesis
}

\author{
Susan L Mills, ${ }^{1}$ Javiera Pumarino, ${ }^{1}$ Nancy Clark, ${ }^{2}$ Simon Carroll, ${ }^{3}$ Sarah Dennis,,${ }^{4,5}$ \\ Sharon Koehn, ${ }^{6,7}$ Tricia Yu, ${ }^{8}$ Connie Davis, ${ }^{2,9}$ Maylene Fong ${ }^{10}$
}

To cite: Mills SL,

Pumarino J, Clark N, et al. Understanding how selfmanagement interventions work for disadvantaged populations living with chronic conditions: protocol for a realist synthesis. BMJ Open 2014;4:e005822. doi:10.1136/ bmjopen-2014-005822

- Prepublication history and additional material is available. To view please visit the journal (http://dx.doi.org/ 10.1136/bmjopen-2014005822).

Received 30 May 2014 Accepted 6 June 2014

\section{CrossMark}

For numbered affiliations see end of article.

Correspondence to Dr Susan L Mills; sue.mills@ubc.ca

\section{ABSTRACT}

Introduction: Self-management programmes are complex interventions aimed at improving the way individuals self-manage chronic conditions, but there are questions about the overall impact of these programmes on disadvantaged populations, in terms of their capacity to engage with and receive the benefits from these initiatives. Given the increased resources being directed towards self-management initiatives, clinicians and policy makers need knowledge on how self-management interventions work for these populations. Most systematic reviews of self-management interventions do not consider the complex interactions between implementation contexts, intervention strategies, and mechanisms that influence how self-management interventions work in real life for disadvantaged groups.

Methods: To address the need for better understanding of these mechanisms and to create context-relevant knowledge, we are conducting a realist synthesis of evidence on self-management interventions for disadvantaged populations living with chronic conditions. The primary research question is: What are the key mechanisms operating in chronic condition selfmanagement interventions among disadvantaged populations? In this protocol, we outline the steps we will take to identify the programme theory for selfmanagement interventions and candidate middle-range theories; to search for evidence in academic and grey literature; to appraise and extract the collected evidence; to synthesise and interpret the findings to generate key context-mechanism-outcome configurations and to disseminate results to relevant stakeholder and to peerreview publications.

Dissemination: Understandings of how chronic conditions self-management interventions work among disadvantaged populations is essential knowledge for clinicians and other decision makers who need to know which programmes they should implement for which groups. Results will also benefit medical researchers who want to direct effort towards current gaps in knowledge in order to advance the self-management field. In addition, the study will make a contribution to the evolving body of knowledge on the realist synthesis method and, in particular, to its application to behaviour change interventions for disadvantaged populations.

\section{INTRODUCTION}

Self-management (SM) programmes are structured interventions that explicitly aim to improve the way individuals self-manage chronic conditions, optimise their health and live well. ${ }^{1}$ These programmes are complex interventions because they often involve multiple topic areas, formats and components (facilitators, participants, family members, technology) that interact over a period of time as the participants move back and forth between intervention processes and day-to-day life. ${ }^{2}$ Moreover, the programmes are often tailored to specific population groups, chronic conditions and/or settings and can produce a range of different results (eg, behaviour, psychosocial, physiological, utilisation outcomes, etc).

SM programmes are increasingly viewed as important healthcare interventions that can help address the complex individual, social and economic impacts of chronic conditions by improving quality of life for patients and reducing the need for intensive chronic condition management services in healthcare systems. ${ }^{3}{ }^{4}$ However, there are serious questions about the overall impact of these programmes on disadvantaged and underserved populations, in terms of their capacity to engage with and receive the benefits from these initiatives. ${ }^{5}$ Studies have frequently shown that these groups have less access, lower rates of participation and much higher rates of attrition in SM programmes than other less vulnerable and more affluent groups. $^{6-8}$ Yet, they have a higher prevalence of chronic conditions, more difficulties managing long-term illness and worse outcomes ${ }^{9}$; all of which place greater strain on healthcare system resources. ${ }^{10}$

In the last decade there has been rapid growth in the number and kind of programmes available, and in the amount of 
funding for SM support initiatives. ${ }^{4}{ }^{11-13}$ Clinicians and policymakers are increasingly faced with the challenges of deciding which kind of SM programme to implement with which populations, necessitating the need for synthesised evidence that can be used in decisionmaking processes. In particular, there is an urgent need for knowledge on how SM interventions work for disadvantaged populations. In the words of a local consultant in Geriatric medicine:

knowledge exchange is better transferred through understanding the context of the person one is living or working with ... However, current reviews of the literature don't address those populations or their realities. (Dr. Chris Rauscher, personal communication, 26 September 2011).

Although studies on SM interventions originally relied on data derived mainly from white, well educated, female populations, ${ }^{14-17}$ the field of SM research has been shifting in recent years in that we now have a substantial number of studies exploring the effectiveness of SM interventions for a range of disadvantaged populations. Not surprisingly, this has led to an increase in the number of systematic reviews on SM interventions tested in populations with a range of different chronic conditions and demographic profiles. ${ }^{18-21}$ Some recently published systematic reviews suggest that SM programmes may be effective and help reduce inequities for disadvantaged populations. ${ }^{22}{ }^{23}$ However, with few exceptions, ${ }^{19}{ }^{24}$ most of the syntheses focus on the effectiveness of SM interventions and do not consider the complex interplay between participants, implementation contexts and processes that can greatly influence intervention results. Contextual factors, such as level of health literacy, culture and economic status influence both SM behaviour and how SM interventions work in real life for disadvantaged groups. ${ }^{6} 81922$ These syntheses also fail to consider the multilevel components of SM interventions or the diversity found in delivery formats, content, facilitators and settings, all of which are necessary considerations in understanding what affects differences in programme outcomes. What is missing, due to the methodological approach of traditional knowledge syntheses, is information on the underlying causal mechanisms that drive programme success or failure, as well as information on how different implementation contexts impact on the efficacy of these mechanisms.

\section{Aims, research questions and objectives}

To address the need for better understanding these underlying mechanisms and context-relevant knowledge, we are conducting a realist synthesis (RS) of evidence on SM interventions for disadvantaged populations living with chronic conditions. The project aims to determine the key features of context (eg, personal characteristics, setting of interventions), intervention (eg, cultural adaptations) and mechanisms (eg, engagement processes, understanding processes) that produce specific outcomes (eg, behaviours, physiological and psychosocial outcomes) for disadvantaged populations. The primary research question guiding this synthesis is: What are the key mechanisms operating in chronic condition SM interventions among disadvantaged populations? Secondary questions include: What contextual features enable or constrain how certain SM interventions work for different disadvantaged populations? What are the key causal mechanisms responsible for particular outcomes? How do SM intervention components and processes trigger mechanisms in particular contexts? How do barriers and enablers influence SM intervention mechanisms and outcomes for disadvantaged populations?

The main objectives of this project are: (1) to develop a programme theory of how SM interventions might succeed for particular disadvantaged groups by triggering specific mechanisms in specific contexts; (2) to develop recommendations for how the findings of the study can be used to assist decision makers in developing and implementing SM interventions in specific populations; (3) to foster and strengthen partnerships among healthcare professionals, policy developers, decision makers and researchers by nurturing multisectorial engagement throughout the research process; (4) to learn from international, national and local knowledge and experience on SM interventions for disadvantaged groups and (5) to identify a research agenda for researchers and funders that addresses gaps that emerge from the synthesis.

\section{Research team}

The project team is comprised of three international researchers (Canada, the USA, the UK and Australia), five local knowledge-users, one information scientist, a research manager, a PhD student and an RS consultant (see table 1).

Knowledge user team members have been actively engaged in all aspects of the project in keeping with an integrated approach to knowledge translation. ${ }^{25}$ The majority of team members have participated in training workshops and based on individual preferences and expertise they have engaged in grant proposal writing and development of literature search and screening strategies. To date, we have benefited greatly from knowledge users' front-line experience and knowledge of SM and disadvantaged populations. In particular, programme managers and clinicians are providing important insights into how SM interventions work in practice which will help us to identify important mechanisms and guide the programme theory development process.

\section{METHODS AND ANALYSIS}

\section{Realist synthesis methodology}

The RS approach is well suited to the specific aims and objectives of our research project because it is designed for synthesising knowledge on multifaceted interventions and provides an approach for considering the 
Table 1 Realist synthesis research team

\begin{tabular}{|c|c|c|}
\hline Team members & Organisation & Role \\
\hline Susan Mills & School of Population and Public Health, The University of British Columbia & Principal investigator \\
\hline Sarah Dennis & $\begin{array}{l}\text { Centre for Primary Health Care and Equity, The University of New South Wales } \\
\text { Clinical and Rehabilitation Science, The University of Sydney }\end{array}$ & Co-investigator \\
\hline Sharon Koehn & $\begin{array}{l}\text { Centre for Healthy Aging, Providence Health Care } \\
\text { Department of Gerontology, Simon Fraser University }\end{array}$ & Co-investigator \\
\hline Nancy Clark & School of Nursing, The University of British Columbia & Co-investigator \\
\hline Connie Davis & $\begin{array}{l}\text { Centre for Collaboration, Motivation and Innovation } \\
\text { Connie L. Davis Health Services }\end{array}$ & Knowledge-user \\
\hline Maylene Fong & Vancouver Community, Home Health Program, Vancouver Coastal Health & Knowledge-user \\
\hline Kelly McQuillen & Primary Health Care and Specialist Services, BC Ministry of Health & Knowledge-user \\
\hline Caryl Harper & Primary Health Care and Specialist Services, BC Ministry of Health & Knowledge-user \\
\hline Chris Rauscher & Geriatric Medicine, Vancouver Coastal Health & Knowledge-user \\
\hline Carole Gillam & Primary Care, Vancouver Coastal Health & Knowledge-user \\
\hline Tricia Yu & Woodward Library, The University of British Columbia & Information scientist \\
\hline Simon Carroll & Department of Sociology, University of Victoria & Methodological consultant \\
\hline Javiera Pumarino & School of Population and Public Health, The University of British Columbia & Research manager \\
\hline
\end{tabular}

complex interplay between individual and social factors that influence intervention processes and beneficial outcomes for disadvantaged populations. ${ }^{26} 27$ Moreover, it values all forms of evidence and enables our research to be grounded in the knowledge and expertise of knowledge users with first-hand experience of the interventions and populations. ${ }^{27}$ The main goal of our RS is to produce an explanatory analysis of how SM interventions work, for which disadvantaged groups, in what circumstances and in what respects. All interventions including SM ones are based on particular understandings of how they work and this needs to be illuminated and tested to see if it reflects the evidence we find. If not, we will generate a revised understanding of how SM interventions work relative to the circumstances and populations of interest. $^{26}$

As described in the seminal work of Pawson, Greenhalgh, Harvey and Walshe, ${ }^{27}$ the RS entails five iterative processes: (1) identifying the programme theory for SM interventions and candidate middle-range theories; (2) searching for evidence; (3) appraising the evidence and extracting data; (4) synthesising and interpreting the findings and (5) disseminating results.

\section{Identifying programme theory and candidate middle-range theories}

To surface a preliminary programme theory about how SM interventions are supposed to work, we will bring our team together for a series of brainstorming sessions. Our aim will be to illuminate the important interactions between different environmental, organisational and personal implementation factors (context-G), and intervention resources that trigger participants' responses (mechanisms-M) to produce changes in behaviour and/or biopsychosocial states (outcomes$\mathrm{O})$, that is, the CMO configurations operating in the

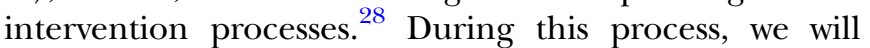
map out the imagined journey that an individual takes over time, starting with accessing the SM programme to participating in group or individual sessions that involve moving back and forth between the programme and daily life settings. We will try to identify all the contextual factors that we feel influence an individual's intervention experience and all the potential short, middle and longer term outcomes of an SM intervention. Throughout these meetings, the goal will be to hypothesise about key relationships between important contextual elements for our populations of interest and intervention implementation, mechanisms mediating intervention resources, and participants' responses and final health outcomes.

The team will also explore potential theories that could provide an explanation for the hypothesised mechanisms of action identified in our brainstorming sessions. ${ }^{29}$ We will try to identify 'candidate middle-range theories' that are concrete enough to provide explanations for what is observed in the evidence and generalisable enough to be applied to a range of different interventions. ${ }^{30}$ We will conduct literature searches for concepts related to SM interventions (concept mining) and for any middle-range theories that might help to illuminate key mechanisms for behaviour change processes in disadvantaged populations using SM interventions (theory mining). After compiling a list of relevant concepts and candidate theories we will map them against the CMO configurations identified in our programme theory brainstorming sessions. Akin to the programme theory, the list of candidate middle-range theories is expected to evolve as the review moves forward; new relevant theories may emerge from the data or we may need to search for new theories that explain newly discovered mechanisms. ${ }^{29} 31$

\section{Searching and screening for evidence}

Subsequently, we will use a purposive sampling strategy to find sufficient and relevant empirical evidence to 
identify important $\mathrm{CMO}$ configurations and test them against the ones in the preliminary programme theory and/or selected candidate middle-range theories. ${ }^{26}$ In addition to a search of the academic peer-reviewed literature, we will conduct a grey literature search to capture a wide array of empirical evidence, which will include theses, dissertations, and evaluation or implementation reports. We feel it is important to capture potentially relevant work being carried out with disadvantaged populations that might not have occurred in environments with research and publication priorities.

The academic and grey literature searches will be conducted by the team under the guidance of two information scientists. The searches will be limited to English language sources, and to documents published after January 2002, because research on SM interventions in disadvantaged populations is a fairly recent development. The inclusion criteria will be SM interventions for adults aged 18 years and over living with one or more of the following chronic conditions: diabetes, hypertension, heart disease, chronic lung disease, depression and other mental health conditions (including mood disorders, anxiety and schizophrenia), rheumatoid arthritis, osteoarthritis, hepatitis C, HIV/AIDS, tuberculosis and/or chronic pain. We selected these conditions either because they have been prioritised by the B.C. Ministry of Health in collaboration with a number of B.C. physician associations and colleges ${ }^{32}$ and/or they have been found to be highly prevalent in disadvantaged populations. ${ }^{33-38}$

We have defined 'disadvantage' as well-established social circumstances that have the potential to negatively influence health and well-being for specific individuals or groups compared to the majority of the population. This includes individuals that have low income, low education, live in rural locations, belong to an ethnic minority or indigenous population, are immigrants and/or aged ( $>65$ years). While the use of these types of categories has limitations (eg, not all members of these groups experience social and/or health inequities), we have chosen these sociodemographic characteristics to define our literature search parameters in order to reflect populations that are more likely to be disadvantaged as outlined by the Campbell and Cochrane Equity Methods Group and the Cochrane Public Health Review Group. ${ }^{39}$

\section{Academic literature}

The academic literature search strategy that we have developed includes search terms related to (1) SM interventions and programmes, (2) disadvantaged populations of interest and (3) selected chronic conditions (see online supplementary file 1). Based on a pilot search in OvidSP MEDLINE that yielded 422 results, the search strategy was revised to take a more broadly encompassing approach by removing the 'chronic disease' concept as an overarching one and making it one of the possible search terms for a specific chronic condition in question. Using the revised search strategy, we will search for citations of primary studies across five standard bibliographic databases: OvidSP MEDLINE, OvidSP EMBASE, EBSCO CINAHL, EBSCO PsycINFO and ProQuest Sociological Abstracts.

We will conduct a two-stage screening process of the academic literature. For this process, we have developed two screening tools to select studies that match our definitions of SM interventions, chronic conditions and disadvantaged populations. The first tool will be applied to the initial screening of abstracts, while the second tool will be used to screen the resulting full papers (see table 2). The tools were piloted on a sample of abstracts and full papers by several team members to ensure that we capture the literature that is most relevant to our research question. The chronic conditions and disadvantaged populations' definitions are the same in both tools. However, in relation to defining SM interventions, the abstract screening tool contains a broad definition since most abstracts have very little detail about the content of the intervention while the full paper screening tool has a more specific definition with specific criteria. We will only include interventions that address one or more of the following life skills: problem-solving, decision-making, resource utilisation, patient-provider relations, taking action, goal setting and/or confidence building mechanisms. This decision was based on a growing consensus in the field that SM interventions differ from patient education strategies because of their patient-centred approach and focus on broader life skills to deal with a range of illness impacts on daily life. ${ }^{40}$

In stage 1, two reviewers will independently review all the retrieved abstracts using the abstract screening tool. The principal investigator (PI) will act as the third reviewer when there are discrepancies between the two primary reviewers. The articles that meet the abstract inclusion criteria will be put through a full paper screening process in stage 2. The PI and another team member will screen all the articles using the full paper screening tool and will resolve discrepancies by consensus.

\section{Grey literature}

Our grey literature search will include: (1) theses and dissertations and (2) evaluation or implementation reports of SM interventions for disadvantaged populations. Our search strategy for theses and dissertations will mimic the academic literature search. We will search in the ProQuest Dissertations \& Theses database, the Theses Canada Portal, and in the DART-Europe E-Theses Portal. The retrieved theses and dissertations will go through a two-stage screening process using the abstract and full text screening tools developed for the academic literature screening.

In collaboration with the research team, a library consultant with expertise in grey literature searches will mine the websites of government health ministries and agencies, health-related research institutes and nongovernment organisations focused on SM of chronic conditions, specific chronic conditions or on health of disadvantaged populations to find implementation and/ 
Table 2 Screening tools for abstract and full paper screening

\begin{tabular}{|c|c|c|}
\hline Criteria & $\begin{array}{l}\text { Abstracts screening } \\
\text { Does the abstract }\end{array}$ & $\begin{array}{l}\text { Full paper screening } \\
\text { Does the full paper }\end{array}$ \\
\hline 1. SM Intervention & $\begin{array}{l}\text { Refer to a SM intervention in adult } \\
\text { populations? } \\
\text { A structured programme, project or initiative } \\
\text { applied at the individual or group level that } \\
\text { explicitly aims to improve the way individuals } \\
\text { self-manage their chronic conditions, optimise } \\
\text { their health and live well }\end{array}$ & $\begin{array}{l}\text { Refer to a SM intervention in adult populations? } \\
\text { An intervention or programme that aims to improve } \\
\text { SM skills and knowledge in adults. Must include at } \\
\text { least one of the following life skills: problem solving, } \\
\text { decision-making, resource utilisation, } \\
\text { patient-provider relations, taking action, goal setting } \\
\text { and/or confidence building mechanisms. Must } \\
\text { involve a patient-centred or empowerment approach } \\
\text { to learning (vs traditional education approach of one } \\
\text { way transmission of information) }\end{array}$ \\
\hline 2. Chronic conditions & \multicolumn{2}{|c|}{$\begin{array}{l}\text { Address at least one chronic condition of interest? } \\
\text { Diabetes, hypertension, heart disease, chronic lung disease, depression and other mental health } \\
\text { conditions (including mood disorders, anxiety and schizophrenia), rheumatoid arthritis, osteoarthritis, } \\
\text { hepatitis C, HIV/AIDS, tuberculosis, chronic pain and/or multimorbidities of these conditions } \\
\text { Include the populations of interest? }\end{array}$} \\
\hline $\begin{array}{l}\text { 3. Disadvantaged } \\
\text { populations }\end{array}$ & $\begin{array}{l}\text { Include the populations of interest? } \\
\text { Aged (>65 years), ethnic minorities, indigenous } \\
\text { immigrant }\end{array}$ & Jeople, rural, low income, low education and/or \\
\hline
\end{tabular}

or evaluation reports on SM interventions. In addition, we will contact researchers and service providers by email and/or phone in an effort to identify and obtain SM programme documents not publicly available on the internet. The list of researchers and service providers will be developed by: (1) identifying contact persons/ programmes included on Canadian websites of government health ministries and agencies focused on SM and/or chronic condition; (2) soliciting suggestions for potential contacts from the PI's research community and (3) putting out a call through the CHAINContact, Help, Advice and Information Network. Further connections will be solicited by means of snowball sampling through these networks. Literature will be limited to digital documents generated since January 2002 in Canada, Australia, the UK, the USA and Europe. We will screen the full text of all the retrieved reports using the full paper screening tool developed for the academic literature.

\section{Extracting data, appraising evidence and synthesising findings}

Once we obtain our final sample of documents from the search and screening processes, descriptive information on each of the articles, theses and reports will be compiled in an Excel spreadsheet. This includes basic information to identify the document (eg, ID, title, first author), information about the study (eg, aim, design, population of interest) and information about the intervention (eg, setting, format, components). We will develop a data extraction form to aid in this process. We expect that this descriptive information will help us to track each article during data synthesis and allow us to group study based on specific population, settings, intervention features, chronic condition elements or other features if required in the analytical process.
Then, we will conduct the data extraction process to obtain evidence to test and complement our preliminary programme theory. Two team members will review each document, annotating them and creating diagrams that describe the observed context, mechanisms, outcomes and CMO configurations using a CMO analysis template. Reviewers will also appraise each document, ranking them as low, medium or high in terms of 'fit for purpose', that is, assessing how useful and relevant the data is to the emerging analysis and how the study will contribute to theory testing. ${ }^{2641}$ Documents will be prioritised for data extraction according to their ranking.

Textual data from the sample documents will be extracted and coded using NVivo V.10 software. Our coding structure will be based on context, mechanism and outcome elements from our programme and candidate middle-range theories (deductive codes) and will be continually updated based on new codes that emerge from the evidence during the data extraction process (inductive codes). The software will allow us to organise the evidence into manageable and searchable pieces of information across CMO categories. This will facilitate conceptual analysis of CMO configurations and allow us to have easy access to direct quotes to present our research findings. NVivo has been previously used in realist syntheses to easily access coded sections of text and use them for analysis, team discussions and manuscripts writing. ${ }^{29}$

As we move through the data extraction process, the team will continually synthesise and interpret emerging findings on CMO configurations within and across studies. We will also continue searching for middle-range theories that can shed light on the complex interactions between the elements in these configurations. These findings will help us reformulate the preliminary programme theory and develop a refined explanation of how SM interventions work for disadvantaged populations. 


\section{DISSEMINATION}

The RS approach will allow us to use the existing body of evidence to produce an explanatory understanding of how SM interventions work for disadvantaged populations and provide insights into how different contexts and intervention strategies interact with individual learning and behaviour change processes. Given that clinicians and policy makers are engaged in our research process, knowledge is being exchanged on an ongoing basis as we all learn together over time. We anticipate that the biggest impact of our collective work will occur as we identify and share important learnings about SM interventions and disadvantaged populations and bring this evolving knowledge into our workplaces and planning processes. Our results will be important for people working in chronic condition management and needing to make decision about which SM programmes they should implement for which groups in which settings. The findings will also be of value for SM intervention developers who need insights into how these kinds of interventions may work for diverse groups. Further, results will benefit medical researchers who want to direct their efforts towards current gaps in SM intervention knowledge for disadvantaged populations in order to advance the SM field. Broader research impacts outside of our team and study jurisdiction may occur when final outcomes of the study are distributed in academic (published manuscripts and presentations) and lay formats (policy briefs, webinars and resource tools).

\section{Author affiliations \\ ${ }^{1}$ School of Population and Public Health, Faculty of Medicine, University of British Columbia, Vancouver, British Columbia, Canada \\ ${ }^{2}$ School of Nursing, University of British Columbia, Vancouver, British Columbia, Canada \\ ${ }^{3}$ Department of Sociology, University of Victoria, Victoria, British Columbia, Canada \\ ${ }^{4}$ Faculty of Health Sciences, Clinical and Rehabilitation Sciences, University of Sydney, Sydney, New South Wales, Australia \\ ${ }^{5}$ Centre for Primary Health Care and Equity, University of New South Wales, Sydney, New South Wales, Australia \\ ${ }^{6}$ Department of Gerontology, Simon Fraser University, Burnaby, British Columbia, Canada \\ ${ }^{7}$ Providence Health Care, Vancouver, British Columbia, Canada \\ ${ }^{8}$ Woodward Library, University of British Columbia, Vancouver, British Columbia, Canada \\ ${ }^{9}$ Centre for Collaboration, Motivation and Innovation, Hope, British Columbia, Canada \\ ${ }^{10}$ Vancouver Community, Home Health Program, Vancouver Coastal Health, Vancouver, British Columbia, Canada}

Acknowledgements The authors would like to thank members of the research team including, Dr Thomas Blakeman, Ms Carole Gillam, Ms Caryl Harper, Ms Kelly McQuillen and Dr Chris Rauscher for their meaningful engagement in the project to date. They would also like to acknowledge $\mathrm{Dr}$ Karen Davison and Dr Justin Jagosh for their contributions in the early stages of study design. Their appreciation is further extended to Dr Beth Snow, Ms Kate Salters and Ms Janaki Jayanthan who assisted in the development of the grant proposal and to the British Columbia Centre of Excellence for Women's Health and School of Population and Public Health for administrative support.

Contributors All authors participated in research design. SLM, SD, NC and JP developed the screening tools. SLM, JP and NC drafted the manuscript. All authors read, provided suggested changes and approved the final manuscript.
Funding This work is supported by the Canadian Institutes of Health Research (FRN: 119802)

\section{Competing interests None.}

Provenance and peer review Not commissioned; peer reviewed for ethical and funding approval prior to submission.

Open Access This is an Open Access article distributed in accordance with the Creative Commons Attribution Non Commercial (CC BY-NC 4.0) license, which permits others to distribute, remix, adapt, build upon this work noncommercially, and license their derivative works on different terms, provided the original work is properly cited and the use is non-commercial. See: http:// creativecommons.org/licenses/by-nc/4.0/

\section{REFERENCES}

1. Mills S, Osborne RH, Brady TJ, et al. The international roundtable on the self-management of chronic conditions. Summary Report: 'Minding the Gap': Building a Framework to Bridge Evidence, Policy and Practice in Self-Management Support for People with Chronic Conditions. Vancouver, BC: British Columbia Centre of Excellence for Women's Health, 2009.

2. Craig P, Dieppe P, Macintyre S, et al. Developing and evaluating complex interventions: the new Medical Research Council guidance. BMJ 2008;337:979-83.

3. Bury M, Pink D. The HSJ debate. Self-management of chronic disease doesn't work. Health Serv J 2005;115:18-19.

4. Rogers A, Kennedy A, Bower P, et al. The United Kingdom expert patients programme: results and implications from a national evaluation. Med J Aust 2008;189:S21-4.

5. Shaw BA, Gallant MP, Riley-Jacome M, et al. Assessing sources of support for diabetes self-care in urban and rural underserved communities. J Community Health 2006;31:393-412.

6. Foster M, Kendall E, Dickson P, et al. Participation and chronic disease self-management: are we risking inequitable resource allocation? Aust J Prim Health 2003;9:132-40.

7. Griffiths C, Motlib J, Azad A, et al. Randomised controlled trial of a lay-led self-management programme for Bangladeshi patients with chronic disease. Br J Gen Pract 2005;55:831-7.

8. Kennedy A, Rogers A, Gately C. Assessing the introduction of the expert patients programme into the NHS: a realistic evaluation of recruitment to a national lay-led self-care initiative. Prim Health Care Res Dev 2005;6:137-48.

9. Nolte E, McKee M. Caring for people with chronic conditions: a health system perspective. Berkshire, UK: McGraw-Hill International, 2008.

10. Douglas KA, Yen LE, Korda RJ, et al. Chronic disease management items in general practice: a population-based study of variation in claims by claimant characteristics. Med J Aust 2011;195:198-202.

11. Jordan JE, Osborne RH. Chronic disease self-management education programs: challenges ahead. Med J Aust 2007;186:84-7.

12. Ory MG, Smith ML, Patton K, et al. Self-management at the tipping point: reaching 100,000 Americans with evidence-based programs. $J$ Am Geriatr Soc 2013;61:821-3.

13. Mills $\mathrm{S}$, Jayanthan J, Carter A, et al. Current trends in self-management support in Canada: a report on self-management-related policies and programs. Vancouver, BC: British Columbia Centre of Excellence for Women's Health, 2013.

14. Bruce B, Lorig K, Laurent D. Participation in patient self-management programs. Arthritis Care Res (Hoboken) 2007;57:851-4.

15. Osborne R, Wilson T, McColl G. Does self-management lead to meaningful and sustainable change? Two-year follow-up of 452 Australians. Proceedings of the Commonwealth Department of Health and Ageing Sharing Health Care Initiative Early Wins Workshop; 2002:9-10.

16. Tattersall R. The expert patient: a new approach to chronic disease management for the twenty-first century. Clin Med 2002;2:227-9.

17. Wright C, Barlow J, Turner A, et al. Self-management training for people with chronic disease: an exploratory study. $\mathrm{Br} \mathrm{J}$ Health Psychol 2003;8:465-76.

18. Chodosh J, Morton SC, Mojica W, et al. Meta-analysis: chronic disease self-management programs for older adults. Ann Intern Med 2005;143:427-38.

19. Eakin EG, Bull SS, Glasgow RE, et al. Reaching those most in need: a review of diabetes self-management interventions in disadvantaged populations. Diabetes Metab Res Rev 2002;18:26-35.

20. Khunti K, Camosso-Stefinovic J, Carey M, et al. Educational interventions for migrant South Asians with type 2 diabetes: a systematic review. Diabet Med 2008;25:985-92. 
21. Sarkisian CA, Brown AF, Norris KC, et al. A systematic review of diabetes self-care interventions for older, African American, or Latino adults. Diabetes Educ 2003;29:467-79.

22. Glazier RH, Bajcar J, Kennie NR, et al. A systematic review of interventions to improve diabetes care in socially disadvantaged populations. Diabetes Care 2006;29:1675-88.

23. McDonald J, Harris E, Kurti L, et al. Action on health inequalities: early intervention and chronic condition self-management. Sydney, Australia: Health Inequalities Research Collaboration, Primary Health Care Network, 2004.

24. Norris SL, Engelgau MM, Narayan KV. Effectiveness of self-management training in type 2 diabetes. A systematic review of randomized controlled trials. Diabetes Care 2001;24:561-87.

25. Wathen $\mathrm{C}$, MacMillan $\mathrm{H}$, Ford-Gilboe $\mathrm{M}$, et al. Knowledge to action: an end-of-grant knowledge translation casebook. Ottawa, ON: Canadian Institutes of Health Research, 2010.

26. Pawson R. Evidence-based policy: a realist perspective. Thousand Oaks, CA: SAGE Publications, 2006.

27. Pawson R, Greenhalgh T, Harvey G, et al. Realist review-a new method of systematic review designed for complex policy interventions. J Health Serv Res Policy 2005;10:21-34.

28. Pawson R. The science of evaluation: a realist manifesto. Thousand Oaks, CA: SAGE Publications, 2013.

29. Wong G. The internet in medical education: a worked example of a realist review. In: Hannes K, Lockwood C, eds. Synthesizing qualitative research: choosing the right approach. Chichester: John Wiley \& Sons, 2012:83-112.

30. Wong G, Westhorp G, Pawson R, et al. Realist synthesis: RAMESES training materials. London: The RAMESES Project, 2013.

31. Jagosh J, Pluye $P$, Wong $G$, et al. Critical reflections on realist review: insights from customizing the methodology to the needs of participatory research assessment. Res Synth Methods 2014;5:131-41.

32. British Columbia Ministry of Health. Primary health care charter: a collaborative approach. 2007.

33. Gallant V, Ogunnaike-Cooke S, McGuire M. Tuberculosis in Canada: 1924-2012. Can Commun Dis Rep 2014;40:99-108.

34. Häfner H, An Der Heiden W. Epidemiology of schizophrenia. Can J Psychiatry 1997;42:139-51.

35. Morgan C, Conway P, Currie C. The relationship between self-reported severe pain and measures of socio-economic disadvantage. Eur J Pain 2011;15:1107-11.

36. Ramage-Morin PL, Gilmour H. Chronic pain at ages 12 to 44 . Health Rep 2010;21:53-61.

37. Canadian AIDS Treatment Information Exchange. HIV in Canada: trends and issues that affect HIV prevention, care, treatment and support. Toronto, ON: Canadian AIDS Treatment Information Exchange, 2009.

38. Public Health Agency of Canada. Epidemiology of acute hepatitis $C$ infection in Canada: results from the Enhanced Hepatitis Strain Surveillance System (EHSSS). Ottawa, ON: Public Health Agency of Canada, 2009

39. Tugwell $\mathrm{P}$, Petticrew M, Kristjansson $\mathrm{E}$, et al. Assessing equity in systematic reviews: realising the recommendations of the Commission on Social Determinants of Health. BMJ 2010;341:873-7.

40. Funnell MM, Tang TS, Anderson RM. From DSME to DSMS: developing empowerment-based diabetes self-management support. Diabetes Spectr 2007;20:221-6.

41. Liu JJ, Davidson E, Bhopal RS, et al. Adapting health promotion interventions to meet the needs of ethnic minority groups: mixed-methods evidence synthesis. Health Technol Assess 2012;16:1-469. 